\title{
UMA ABORDAGEM INTERVALAR PARA A CARACTERIZAÇÃO DE INTERVALOS DE DISPARO EM REDES DE PETRI TEMPORAIS
}

\author{
Evangivaldo A. Lima* \\ Ricardo Lüders ${ }^{\dagger}$ \\ evanlima@uneb.br \\ luders@utfpr.edu.br \\ Luis Allan Künzle ${ }^{\ddagger}$ \\ kunzleeinf.ufpr.br \\ *Departamento de Ciências Exatas e da Terra - Universidade do Estado da Bahia \\ Rua Silveira Martins, 2555 \\ 41195-001 Salvador, BA, Brasil \\ ${ }^{\dagger}$ CPGEI - Universidade Tecnológica Federal do Paraná \\ Av. Sete de Setembro 3165 \\ 80230-901 Curitiba, PR, Brasil \\ ${ }^{\ddagger}$ Departamento de Informática - Universidade Federal do Paraná \\ Rua Cel. Francisco H. dos Santos, 100 C. P. 19081 \\ 81531-980, Curitiba, PR, Brasil
}

\begin{abstract}
This paper deals with time Petri nets where a time interval is associated to each transition. Time Petri nets are widely used for modeling and analysis of real-time systems. Although there are several techniques for time Petri net analysis, this paper presents an interval analysis using a global time approach where an algebraic model is proposed for computing of intervals. For a particular class of time Petri nets, this model assumes an interval linear equation form which allows to compute intervals for a firing sequence as well as performance measures for timed systems. By using a redution method for time Petri nets this model assumes a matricial form that can be applied to a broad class of Time Petri nets. Furthermore, this approach is illustrated by computing basic performance metrics for a communication protocol.
\end{abstract}

Artigo submetido em $07 / 07 / 2007$

1a. Revisão em 01/05/2008

2a. Revisão em 29/09/2008

Aceito sob recomendação do Editor Associado

Prof. José Reinaldo Silva
KEYWORDS: Time Petri Nets, Interval Analysis, Discrete Event Systems

\section{RESUMO}

As redes de Petri temporais são uma das extensões das redes de Petri convencionais para modelagem e análise de sistemas a eventos discretos temporizados. A cada transição da rede é associado um intervalo de tempo, o qual delimita o instante de disparo da transição. A análise das redes de Petri temporais tem sido feita por métodos enumerativos que relacionam todos os estados alcançáveis da rede. Neste artigo, usando recursos da álgebra intervalar e utilizando o conceito de classe de estados, é apresentada uma abordagem para resolver problemas relacionados à caracterização de intervalos de tempo de disparo em redes de Petri temporais. A partir de uma expressão algébrica, desenvolvida para cálculo de intervalos de tempo entre duas classes de estados consecutivas, obtém-se uma equação intervalar que possibilita o cálculo de intervalos de tempo entre duas classes de estados quaisquer. Assim, é possível calcular o intervalo de tempo para ocorrência de uma 
seqüência de eventos. Utilizando um método de redução da rede, a equação intervalar assume a forma matricial e, com isso, amplia a classe das redes de Petri temporais à qual a equação intervalar é aplicável. Por fim, uma aplicação explorando as potencialidades da equação intervalar no cálculo de medidas características de um sistema a eventos discretos temporizados é apresentada.

PALAVRAS-CHAVE: Redes de Petri Temporais, Análise Intervalar, Sistemas a Eventos Discretos

\section{INTRODUÇÃO}

As redes de Petri são um formalismo para modelagem e análise de sistemas a eventos discretos (SED), principalmente em sistemas com com alto grau de concorrência (Murata, 1989). Tais redes são bastante usadas na descrição do comportamento causal dos sistemas, pois esta é uma forma confiável para ordenar a ocorrência dos eventos. Na prática, porém, o instante de ocorrência dos eventos é tão importante quanto sua relação de ordem causal. Fatores de desempenho dos sistemas, como o instante de ocorrência de eventos e tempo de ciclo de uma operação, têm gerado uma preocupação cada vez maior com questões temporais (Aura and Lilius, 1996).

As redes de Petri com informação a respeito do instante de ocorrência dos eventos, foram introduzidas independentemente por Ramchandani (1974) e Merlin (1974). Desde então, um grande número de diferentes extensões temporais para redes de Petri têm sido propostas na literatura, cada uma delas sendo usada em uma aplicação específica. Geralmente, as extensões se diferenciam em aspectos como: forma de temporização, localização da restrição temporal e propriedade da restrição (Cerone and Maggiolo-Schettini, 1999). Dentre as extensões temporais para as redes de Petri, destacam-se as Redes de Petri Temporais $(R P T s)$, que permitem que transições, lugares ou marcas carreguem informações temporais (Aura and Lilius, 1996). Nas redes temporais, a informação do tempo é representada por um intervalo de tempo, o que torna esse tipo de rede mais adequada para expressar a maioria dos requisitos temporais de sistemas reais (Bucci and Vicario, 1995; Montano et al., 2000).

$\mathrm{Na}$ análise das RPTs, cada estado é composto por uma marcação e uma informação a respeito do possível instante de disparo das transições. Devido à natureza contínua do tempo, a cada instante gera-se um estado diferente, tornando tal análise inviável por causa do elevado número de estados, mesmo para uma rede simples. Para contornar este problema, os estados foram agrupados em classes e os possíveis instantes de disparo das transições são apresentados como conjuntos de intervalos de tempo (Berthomieu and Diaz,
1991). Variações da análise baseada em classes de estados têm sido apresentadas na literatura, propiciando novas alternativas para análise das RPTs (Yoneda and Schlingloff, 1997; Vicario, 2001; Lilius, 1998).

Nos últimos anos, novas ferramentas matemáticas têm sido utilizadas para explorar resultados algébricos das extensões temporais das redes de Petri. Considerando uma classe particular das redes de Petri temporizadas como modelo de um sistema a eventos discretos temporizado, em Cohen (2001) é mostrado que tal sistema pode ser tratado como um sistema linear, do ponto de vista da álgebra de dióides. Já em Jaulin et al. (1999) é usada a Álgebra Intervalar na estimação de datas de ocorrência em um grafo de eventos temporizados. Em Lima et al. (2006) é apresentado o uso da álgebra intervalar como ferramenta matemática para análise de uma $R P T$. Neste último trabalho, os autores expressam a dinâmica dos intervalos de ocorrência dos eventos por meio de uma equação linear intervalar. Porém, o modelo é aplicável a uma classe restrita das RPTS denominadas redes não persistentes.

Neste artigo, é apresentada uma abordagem intervalar que caracteriza os intervalos de tempo de disparo em RPTs. Usando o conceito de classe de estados, análise de tempo global e regras de redução para uma $R P T$, a abordagem possibilita a estimação de intervalos de tempo de disparo de transições habilitadas em uma classe de estados alcançável pelo disparo de uma seqüência de transições. Neste caso, pode-se obter respostas a questões como: intervalo de tempo para realização de tarefas, verificação de tempo da tarefa, mínimo e máximo tempo entre disparos de transições.

Este artigo está estruturado da seguinte forma. Na Seção 2, é feita uma revisão sucinta de algumas operações da álgebra intervalar. Na Seção 3, são apresentadas as redes de Petri temporais. A dinâmica das RPTs, usando tempo global, é apresentada na Seção 4. Partindo-se de uma expressão algébrica para avaliar classes sucessoras, obtém-se na Seção 5 a estimação de intervalos de tempo de disparo em uma classe de estados qualquer de uma rede de Petri temporal. $\mathrm{Na}$ Seção 6, é apresentado um conjunto de regras para redução de uma rede de Petri temporal. Os resultados desta seção são utilizados na Seção 7 para aplicar a equação intervalar a uma classe mais ampla de redes de Petri temporais. Na Seção 8, é feito um estudo de caso para um sistema temporizado e, concluindo o artigo, as considerações finais são apresentadas na Seção 9.

\section{2 ÁLGEBRA INTERVALAR}

Neste seção, serão definidos alguns conceitos e operações básicas sobre intervalos, cujos detalhes podem ser encontrados em Moore (1995), Jaulin et al. (1995) e 
Hansen and Walster (2004).

Um intervalo $\mathbf{x}=[\underline{x}, \bar{x}]$ é definido como sendo o conjunto $\{x \in \mathbb{R} \mid \underline{x} \leq x \leq \bar{x}\}$ sendo, $\underline{x}$ e $\bar{x}$ denominados limites inferior e superior do intervalo $\mathbf{x}$, respectivamente. Neste caso, o conjunto dos intervalos reais é denotado $\mathbb{I R}$.

A interseção de dois intervalos $\mathbf{x}$ e $\mathbf{y}$ tais que $\mathbf{x} \cap \mathbf{y} \neq \emptyset$ é definida como:

$\mathbf{x} \cap \mathbf{y}=[\max \{\underline{x}, \underline{y}\}, \min \{\bar{x}, \bar{y}\}]$.

A união intervalar de dois intervalos $\mathbf{x}$ e $\mathbf{y}$, denotada por $\sqcup$, é o menor intervalo que contenha $\mathbf{x}$ e $\mathbf{y}$, ou seja,

$\mathbf{x} \sqcup \mathbf{y}=[\mathbf{x} \cup \mathbf{y}]=[\min \{\underline{x}, \underline{y}\}, \max \{\bar{x}, \bar{y}\}]$

\section{Relação de Ordem}

Há na literatura vários tipos de relações de ordem definidas sobre $\mathbb{I} \mathbb{R}$ (Moore, 1995; Jaulin et al., 1995; Hansen and Walster, 2004). Neste trabalho, adota-se:

$\mathbf{x}<\mathbf{y} \Longleftrightarrow \bar{x}<y$

$\mathbf{x} \leq \mathbf{y} \Longleftrightarrow \underline{x} \leq \bar{y}, \bar{x} \leq \bar{y}$

$\mathbf{x} \subseteq \mathbf{y} \Longleftrightarrow \underline{x} \geq \underline{y}, \quad \bar{x} \leq \bar{y}$

\section{Operações básicas}

$$
\begin{aligned}
& \mathbf{x}+\mathbf{y}=[\underline{x}+\underline{y}, \bar{x}+\bar{y}] \\
& \mathbf{x}-\mathbf{y}=[\underline{\bar{y}}, \bar{x}-\underline{y}]
\end{aligned}
$$

Um vetor intervalar $\mathbf{x} \in \mathbb{R}^{n}$ é um vetor cujas $n$ componentes são intervalos em $\mathbb{I R}$.

Uma extensão da operação diferença entre intervalos é denominada subtração dependente (Moore, 1995) e definida por:

$$
\mathbf{x} \ominus \mathbf{y}=[\underline{x}-\underline{y}, \bar{x}-\bar{y}]
$$

Observa-se que tal operação pode não resultar em um intervalo clássico.

\section{Equações Lineares Intervalares}

São expressões da forma

$$
\mathbf{A x}=\mathbf{b},
$$

com $\mathbf{A} \in \mathbb{R}^{m \times n}, \mathbf{b} \in \mathbb{R}^{m}, \mathrm{x} \in \mathbb{R}^{n}$. A Equação 1 pode ser tratada como a família de todos os sistemas de equações lineares do tipo

$$
\mathrm{Ax}=\mathrm{b}
$$

sendo $\mathrm{A} \in \mathbb{R}^{m \times n}, \mathrm{~b} \in \mathbb{R}^{m}$ tal que $\mathrm{A} \in \mathbf{A}$ e $\mathrm{b} \in \mathbf{b}$.

O conjunto solução para a Equação 1, denominado solução geral (united solution set), é o conjunto

$$
\mathcal{S}_{g}=\left\{\mathrm{x} \in \mathbb{R}^{n} \mid \exists \mathrm{A} \in \mathbf{A}, \exists \mathrm{b} \in \mathbf{b} \text { tal que } \mathrm{Ax}=\mathrm{b}\right\} .
$$

$\mathcal{S}_{g}$ é o conjunto de todas as soluções possíveis para a Equação 1.

Para ilustrar a solução dada pelo conjunto definido por 3, segue o seguinte exemplo:

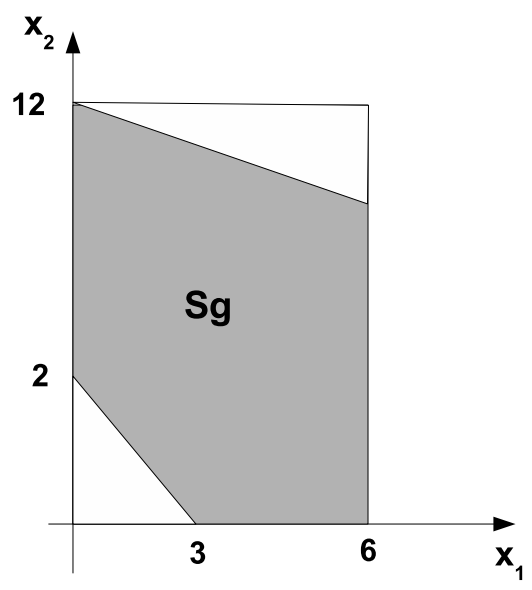

Figura 1: Conjunto solução $\mathcal{S}_{g}$

$$
\begin{aligned}
& {[2,3] \mathrm{x}_{1}+[0,1] \mathrm{x}_{2}=[0,12]} \\
& {[1,2] \mathrm{x}_{1}+[2,3] \mathrm{x}_{2}=[6,24]}
\end{aligned}
$$

Sendo $\mathrm{x}_{1} \geq 0$ e $\mathrm{x}_{2} \geq 0$ as equações acima podem ser reescritas como:

$$
\begin{aligned}
& {\left[2 \mathrm{x}_{1}, 3 \mathrm{x}_{1}+\mathrm{x}_{2}\right]=[0,12]} \\
& {\left[\mathrm{x}_{1}+2 \mathrm{x}_{2}, 2 \mathrm{x}_{1}+3 \mathrm{x}_{2}\right]=[6,24]}
\end{aligned}
$$

A solução $\mathcal{S}_{g}$, neste exemplo, está ilustrada na Figura 1. Uma solução intervalar para representar $\mathcal{S}_{g}$ poderia ser o menor vetor intervalar que contenha $\mathcal{S}_{g}$. Para o exemplo da Figura 1 , este vetor é dado por

$$
\mathbf{x}=\left(\begin{array}{c}
{[0,6]} \\
{[0,12]}
\end{array}\right)
$$

Nota-se que $\mathbf{x}$ contém pontos que não estão em $\mathcal{S}_{g}$, porém, todo ponto de $\mathcal{S}_{g}$ está em $\mathbf{x}$.

\section{REDES DE PETRI TEMPORAIS}

As RPTs são uma das mais eficientes ferramentas para modelagem de sistemas concorrentes com restrições de tempo associadas à ocorrência dos eventos. Nas RPTs, cada ocorrência de evento é associada a um intervalo de tempo. Os limites inferior e superior desse intervalo de tempo determinam o mínimo e o máximo instantes para a ocorrência do evento. Com isso, torna-se possível modelar restrições de tempo onde o instante exato da ocorrência é 
desconhecido. Em sistemas reais esta situação é bastante comum pois, na prática, especificam-se limites de tempo quando se desconhece seu valor exato. $\mathrm{O}$ intervalo que delimita o instante de ocorrência de cada evento será denominado de domínio de tempo dessa ocorrência.

Definição 3.1 (Domínio de Tempo) - Seja $\mathbb{R}$ o conjunto dos intervalos reais. Denota-se por $\mathbf{I}=\mathbf{i}_{1} \times \mathbf{i}_{2} \times \ldots \times \mathbf{i}_{n}$ o vetor intervalar domínio de tempo, cujos elementos são intervalos fechados tais que

$$
\mathbf{i}_{k}=[a, b]=\{i \in \mathbb{R} \mid a \leq i \leq b\} k=1, \ldots, n
$$

O domínio de tempo é um vetor cujas coordenadas são intervalos de tempo. O domínio varia seus elementos conforme o estado em que a rede se encontra. Quando o intervalo $\mathbf{i}_{k}$ apresentar $a=b$, será chamado de intervalo degenerado ou intervalo com tempo de disparo definido. $\mathrm{O}$ elemento nulo 0 significa um evento desabilitado. Assim, diferencia-se o intervalo degenerado $[0,0]$ do elemento nulo 0 .

Definição 3.2 (Rede de Petri Temporal) - Formalmente, uma rede de Petri temporal é uma sêxtupla $R P T=$ $\left(P, T\right.$, Pre, Post, FI, $\left.M_{0}\right)$.

Sendo,

$P$ o conjunto de lugares;

$T$ o conjunto de eventos (ou transições);

$P \cap T=\emptyset$;

Pre $: P \times T \longrightarrow \mathbb{N}$, a função incidência de entrada, sendo $\mathbb{N}$ o conjunto dos números naturais (peso dos arcos de entrada das transições)

Pos : $T \times P \longrightarrow \mathbb{N}$, a função incidência de saída, sendo $\mathbb{N}$ o conjunto dos números naturais (peso dos arcos de saída das transições)

$F I: T \longrightarrow \mathbb{R}$, a função intervalo estático;

$M_{0}$, a marcação inicial da $R P T$.

A função $F I$ associa a cada transição $t_{i} \in T$ da rede um intervalo de tempo $\mathbf{i}^{s}\left(t_{i}\right)=\left[\delta^{s}, \Delta^{s}\right]$, denominado domínio de tempo estático da transição, sendo o vetor $\mathbf{I}^{s}$ é denominado de vetor domínio de tempo estático. Os limites $\delta^{s}$ e $\Delta^{s}$ são chamados limite inferior de ocorrência $(L I O)$ e limite superior de ocorrência $(L S O)$, respectivamente.

Definição 3.3 (Habilitação de uma Transição) - Uma transição $t \in T$ em uma $R P T$ é dita habilitada pela marcação $\mathrm{M}$ se, e somente se, $\mathrm{M} \geq \operatorname{Pre}(t)$, sendo $\mathrm{M} \mathrm{o}$ vetor marcação e $\operatorname{Pre}(t)$ a coluna $t$ da matriz incidência de entrada da rede (Murata, 1989).
Nas redes de Petri convencionais, uma transição habilitada significa que o evento a ela associado pode ocorrer a qualquer instante e sua ocorrência é caracterizada pelo disparo da transição. Porém, nas RPTs, o fato de uma transição $t$ estar habilitada, não significa necessariamente que ela será disparada. Primeiro, é preciso que a transição permaneça habilitada por um tempo mínimo igual ao $L I O$, a partir do qual ela pode ser disparada. Por outro lado, caso a transição permaneça habilitada até o instante igual ao $L S O$, então necessariamente deverá ser disparada. Dessa forma, comparada às redes de Petri convencionais, nas RPTs a indeterminação de uma ocorrência é reduzida pela obrigatoriedade do disparo da transição. Portanto, para uma descrição completa do estado de uma $R P T$ é necessária a marcação atual da rede e uma informação a respeito do tempo de habilitação das transições. O conjunto formado por todas as transições habilitadas pela marcação M será denotado $\mathcal{H}$. Outro fator importante na habilitação das RPTs é a fato de que, se uma transição permanece habilitada após seu disparo, então sua temporização é deve ser reinicializada, inviabilizando o conceito de múltipla habilitação (Berthomieu and Diaz, 1991).

Definição 3.4 (Estado) - O estado de um $R P T$ é caracterizado pelo par $E=(\mathrm{M}, \mathrm{I})$, sendo M o vetor marcação da rede e $I \in \mathbb{R}^{n}$ o vetor tempo de habilitação das transições habilitadas neste estado.

Assim,

$$
\begin{aligned}
& \mathcal{H} \longrightarrow \mathrm{I} \\
& t \longmapsto \mathrm{i}(t)
\end{aligned}
$$

a informação temporal de cada transição habilitada $t \in \mathcal{H}$ tem um domínio intervalar. O estado inicial das $R P T$ é $E_{0}=\left(\mathrm{M}_{0}, \mathrm{I}_{0}\right)$, sendo $\mathrm{I}_{0} \in \mathbf{I}^{s}$ o vetor de elementos racionais correspondente aos instantes em que as transições se tornaram habilitadas pela marcação $\mathrm{M}_{0}$.

A mudança de estado em uma $R P T$ pode ocorrer devido à passagem do tempo (sem disparo de transição) ou devido ao disparo de uma transição (sem acréscimo no tempo). Dessa forma, múltiplas possibilidades de estados podem ser explicitadas. Geralmente, a mudança do estado pelo tempo é aplicada em verificação formal, seja combinado com lógica temporal (Yoneda and Schlingloff, 1997; Gardey et al., 2005), com processo temporal e unfolding (Aura and Lilius, 1996; Lilius, 1998) ou utilizando a matriz de intervalos DBM (Difference Bound Matrix) (Vicario, 2001; Gardey et al., 2005). Na mudança de estado devido ao disparo de uma transição, a marcação e os tempos das transições habilitadas no novo estado são atualizados. Porém, devido à natureza contínua do tempo, inúmeros estados podem 
ser gerados dentro do domínio do tempo de disparo de cada transição habilitada, o que torna o modelo intratável, sob o ponto de vista computacional. Nesse sentido, o conceito de Classe de Estados, introduzido em Berthomieu and Menasche (1982) e ampliado em Berthomieu and Diaz (1991), contribuiu decisivamente para o desenvolvimento das técnicas de análise para as RPTs.

Definição 3.5 (Classe de Estados) - Uma classe de estados em uma $R P T$ é definida pelo par $C E=\langle\mathrm{M}, \mathbf{I}\rangle$, sendo

- M a marcação da rede

- I o vetor domínio de tempo das transições habilitadas por $\mathrm{M}$, também chamado domínio dinâmico. Para a classe inicial $\mathbf{I}_{0} \subseteq \mathbf{I}^{s}$.

Para ilustrar os conceitos de estado e classe de estados, na Figura $2 a$ é mostrada uma $R P T$ com duas transições $t_{1}$ e $t_{2}$, cujos domínios de tempo são $[1,2]$ e $[2,3]$, respectivamente. Contando o tempo de disparo a partir do instante em que a transição é habilitada pela marcação e considerando que a transição $t_{1}$ tornou-se habilitada no instante 0 , o estado inicial da rede é $E_{0}=\left\langle\mathrm{M}_{0}, 0\right\rangle$, sendo $\mathrm{M}_{0}=\left[\begin{array}{ll}2 & 0\end{array}\right]^{T}$. Considerando a mudança de estado pelo disparo da única transição habilitada, a mudança da marcação inicial ocorre apenas a partir de 1 unidade de tempo, devido ao LIO. Caso a transição $t_{1}$ mantenha-se habilitada até 2 unidades de tempo, então deverá ser disparada, já que esse instante corresponde ao $L S O$ da transição $t_{1}$. Portanto, existe um número infinito de estados desde o instante 1 até o instante 2 , conforme a Figura $2 b$. Na definição de classe de estados, entretanto, considera-se apenas o intervalo de tempo no qual há possibilidade de disparo das transições. Portanto, para a rede mostrada na Figura $2 a$, a classe inicial seria $C E_{0}=\left\langle\mathrm{M}_{0}, \mathbf{I}_{0}\right\rangle$, sendo $\mathbf{I}_{0}=\left(\begin{array}{c}{[1,2]} \\ 0\end{array}\right)$. O intervalo $[1,2]$ representa o domínio de tempo de disparo da transição $t_{1}$.

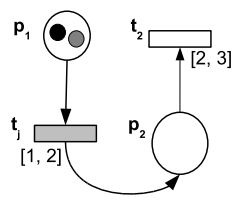

(a)

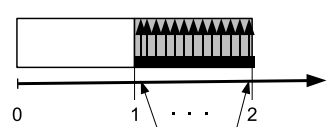

Estados

(b)
Apesar das vantagens em termos de uma representação mais compacta, a análise de uma rede de Petri temporal via classes de estados apresenta algumas limitações, principalmente quanto a questões de alcançabilidade ou do conjunto de estados finais. Diversos métodos que buscam superar as limitações das classes de estado têm surgido nos últimos anos, principalmente para a verificação formal de modelos. Muitos destes trabalhos, mesmo usando as classes de estados propostas em Berthomieu and Menasche (1982) e Berthomieu and Diaz (1991), procuram superar suas limitações pela modificação na forma de tratar o tempo, como em Tsai et al. (1995), Vicario (2001) e Wang and Deng (2000).

Existem ainda na literatura trabalhos que modelam sistemas temporais como autômatos temporizados (Alur and Dill, 1994). Através de uma estrutura de clock, definida como uma região ou zona de clock, os estados são compactados formando regiões de estados, geralmente na forma de poliedros convexos. Embora os conceitos pareçam semelhantes, a classe de estados nas RPTs é definida a partir das transições que permanecem habilitadas numa dada marcação durante um intervalo de tempo definido pelos intervalos estáticos das transições.

\section{DINÂMICA DAS RPT COM TEMPO GLOBAL}

Com o disparo de uma transição, há a necessidade de atualização dos domínios de tempo da nova classe de estados da rede. Existem duas abordagens diferentes quanto ao tratamento do tempo na atualização das classes de estados: a primeira trata o tempo de forma relativa ao instante em que a transição foi habilitada (Berthomieu and Diaz, 1991; Popova-Zeugmann, 1998), já a segunda trata o tempo de forma global, ou seja, os domínios são relativos ao início de execução da rede (Wang and Deng, 2000; Xu et al., 2002; Lima et al., 2005). Considerando a semântica do tempo global, a dinâmica das RPTs consiste em explicitar todas as classes de estados alcançáveis a partir da classe inicial.

Nas RPTs, uma transição habilitada pela marcação em uma classe de estados, não necessariamente será disparada nessa classe. Para que uma transição habilitada seja disparável é preciso que ela atenda às condições temporais definidas a seguir.

Definição 4.1 (Transição Disparável) Uma transição $t_{j} \in$ $T$ habilitada na classe de estados $C E=(\mathrm{M}, \mathbf{I})$ de uma $R P T$ é disparável se, e somente se,

- o tempo de habilitação é, no mínimo, igual a $L I O\left(t_{j}\right)$ $\mathrm{e}$ 
- $L I O\left(t_{j}\right)$ é menor ou igual aos demais $L S O$ das transições habilitadas na classe.

Portanto, se a transição $t_{j}$ for disparável, então terá um intervalo de disparo $\mathbf{i}^{d}=\left[\delta^{d}, \Delta^{d}\right]$ tal que

$$
\Delta^{d} \leq \min \{\Delta(t)\}
$$

sendo $t \in \mathcal{H}$.

O conjunto das transições disparáveis em uma classe é denominado de $\mathcal{D}$.

Definição 4.2 (Transição Persistente) Uma transição é dita persistente se, uma vez habilitada, apenas seu disparo poderá desabilitá-la.

Uma transição que permanece habilitada após o disparo de uma outra é chamada herdada.

\subsection{Mudança de Classe de Estados}

Com o disparo de uma transição $t_{j}$, os valores para a marcação e dos domínios de tempo da nova classe são atualizados. Seja, por exemplo, $C E_{1}=\left\langle M_{1}, \mathbf{I}_{1}\right\rangle$ uma classe de estados de uma $R P T$ alcançável a partir da classe $C E_{0}=$ $\left\langle M_{0}, \mathbf{I}_{0}\right\rangle$ pelo disparo da transição $t_{j}$. Sendo $\mathbf{i}_{0}^{d}=\left[\delta^{d}, \Delta^{d}\right] \mathrm{o}$ intervalo de disparo de $t_{j}$ na classe $C E_{0}$, então a classe $C E_{1}$ é assim caracterizada:

1. A nova marcação resulta da remoção e adição de marcas nos lugares de entrada e saída da transição $t_{j}$,

$$
\mathrm{M}_{1}=\mathrm{M}_{0}+\operatorname{Post}\left(t_{j}\right)-\operatorname{Pre}\left(t_{j}\right)
$$

2. O vetor domínio de tempo dinâmico é atualizado de forma diferente para transições que já estavam habilitadas (transições persistentes) e para transições que foram habilitadas após o disparo de $t_{j}$ (transições recém-habilitadas), ou seja,

$$
\begin{aligned}
& \mathbf{I}_{1}(t)=\left[\max \left\{\delta^{d}, \delta_{0}(t)\right\}, \Delta_{0}(t)\right] t \in \mathcal{P}_{1} \\
& \mathbf{I}_{1}(t)=\mathbf{i}^{d}+\mathbf{I}^{s}(t) \quad t \in \mathcal{N}_{1} .
\end{aligned}
$$

sendo $\mathcal{N}_{1}$ o conjunto das transições recém-habilitadas e $\mathcal{P}_{1}$ o conjunto das transições persistentes na classe $C E_{1}$.

3. As transições desabilitadas por $M_{1}$ têm domínios de tempo nulos.

$\mathrm{Na}$ Equação (6), as transições persistentes podem ter apenas seu limite inferior de disparo alterado, ou seja,

$$
\begin{aligned}
& \delta^{d} \leq \delta_{0}(t), \text { tem-se } \max \left\{\delta^{d}, \delta_{0}(t)\right\}=\delta_{0}(t) \text { e } \mathbf{I}_{1}(t)=\mathbf{I}_{0}(t) \\
& \delta^{d}>\delta_{0}(t), \text { tem-se } \max \left\{\delta^{d}, \delta_{0}(t)\right\}=\delta^{d}(t) \text { e } \mathbf{I}_{1}(t) \subset \mathbf{I}_{0}(t)
\end{aligned}
$$

Assim, mantendo-se tal limite inalterado, a Equação (6) pode ser rearranjada como:

$$
\mathbf{I}_{1}(t)=\left\{\begin{array}{lr}
{\left[\delta_{0}(t), \Delta_{0}(t)\right]=\mathbf{I}_{0}(t)} & t \in \mathcal{P}_{1} \\
\mathbf{i}^{d}+\mathbf{I}^{s}(t) & t \in \mathcal{N}_{1}
\end{array}\right.
$$

Em Lima et al. (2006), mostra-se que Equação 7 assume a seguinte forma vetorial:

$$
\mathbf{I}_{1}=\left(\mathbf{i}^{d} \mathrm{u}+\mathbf{I}^{s}\right) \mathrm{n}_{1}+\mathbf{I}_{0} \mathrm{p}_{1}
$$

sendo:

$\mathbf{I}_{1} \in \mathbb{R}^{n}$ o vetor domínio de tempo dinâmico;

$\mathbf{I}^{s} \in \mathbb{R}^{n}$ o vetor domínio de tempo estático;

$\mathbf{i}^{d} \in \mathbb{R}$ o intervalo de disparo de $t_{j} ;$

$\mathrm{n}_{1} \in\{0,1\}^{n}$ o vetor das transições recém habilitadas;

$\mathrm{p}_{1} \in\{0,1\}^{n}$ o vetor das transições persistentes;

$\mathrm{h}_{1}=\mathrm{n}_{1}+\mathrm{p}_{1}$ o vetor das transições habilitadas; $\mathrm{u} \in\{1\}^{n}$.

Os vetores $\mathrm{p}, \mathrm{n}$ e $\mathrm{h}$, são vetores binários de ordem igual ao número de transições da rede, sendo o produto entre dois vetores definido elemento a elemento. Para o estado inicial, o vetor domínio de tempo é dado por $\mathbf{I}_{0}=\mathbf{I}^{s} \mathrm{~h}_{0}$, sendo $\mathrm{h}_{0} \mathrm{O}$ vetor das transições inicialmente habilitadas.

$\mathrm{O}$ vetor das transições recém habilitadas é obtido com o auxílio de um vetor coluna $\mathrm{q}$, denominado vetor de disparo, que possui zeros em todas as $n$ posições, exceto na posição correspondente à da transição a ser disparada. Assim,

$$
\mathrm{n}_{i+1}=\mathrm{h}_{i+1}-\left(\mathrm{h}_{i}-\mathrm{q}\right) i \geq 0
$$

Generalizando as Equações (5) e (8), têm-se:

$$
\left\{\begin{array}{l}
\mathrm{M}_{k+1}=\mathrm{M}_{k}+\operatorname{Post}(t)-\operatorname{Pre}(t) \\
\mathbf{I}_{k+1}=\left(\mathbf{I}^{d} \mathrm{u}+\mathbf{I}^{s}\right) \mathrm{n}_{k+1}+\mathbf{I}_{k} \mathrm{p}_{k+1}
\end{array}\right.
$$

A Equação 9 permite caracterizar recursivamente uma classe de estados de uma RPT sem que seja necessário executar a rede.

Exemplo 4.1 Baseado na $R P T$ mostrada na Figura 3 e aplicando a equação 9 , determina-se o intervalo de tempo necessário para que a transição $t_{4}$ possa ser disparada.

As matrizes de entrada e saída da rede e o vetor domínio estático, são dados a seguir: 


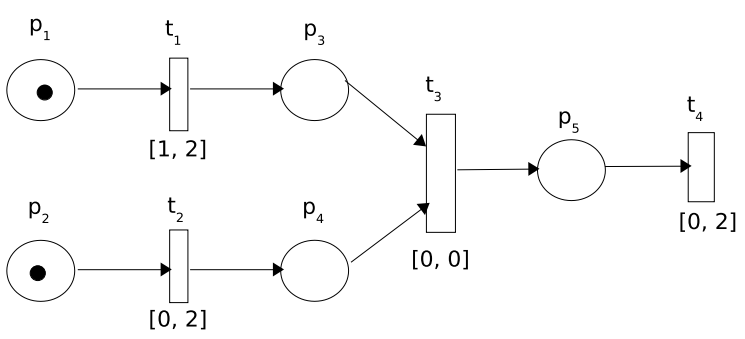

Figura 3: $R P T$ para o Exemplo 4.1

Pre $=\left(\begin{array}{llll}1 & 0 & 0 & 0 \\ 0 & 1 & 0 & 0 \\ 0 & 0 & 1 & 0 \\ 0 & 0 & 1 & 0 \\ 0 & 0 & 0 & 1\end{array}\right)$ Pos $=\left(\begin{array}{llll}0 & 0 & 0 & 0 \\ 0 & 0 & 0 & 0 \\ 1 & 0 & 0 & 0 \\ 0 & 1 & 0 & 0 \\ 0 & 0 & 1 & 0\end{array}\right)$

$\mathbf{I}^{s}=([1,2][0,2][0,0][0,2])^{T}$

- A classe inicial $C E_{0}=\left\langle\mathrm{M}_{0}, \mathbf{I}_{0}\right\rangle$ é caracterizado como segue:

$$
\begin{aligned}
& \mathrm{M}_{0}=\left[\begin{array}{lllll}
1 & 1 & 0 & 0 & 0
\end{array}\right]^{T} \\
& \mathrm{M}_{0} \geq \operatorname{Pre}\left(t_{1}\right), \mathrm{M}_{0} \geq \operatorname{Pre}\left(t_{2}\right) .
\end{aligned}
$$

Assim,

$$
\begin{aligned}
& \mathrm{h}_{0}=\left[\begin{array}{llll}
1 & 1 & 0 & 0
\end{array}\right]^{T} . \\
& \mathbf{I}_{0}=\mathbf{I}^{s} \mathrm{~h}_{0}=\left(\left[\begin{array}{llll}
1,2] & {[0,2]} & 0 & 0
\end{array}\right)^{T}\right.
\end{aligned}
$$

Portanto, na classe inicial as transições $t_{1}$ e $t_{2}$ estão habilitadas e ambas são disparáveis, pois satisfazem às condições da Definição 4.1.

- Representando o disparo da transição $t_{2}$ através do vetor de disparo $\mathrm{q}=\left[\begin{array}{llll}0 & 1 & 0 & 0\end{array}\right]^{T}$ no intervalo $\mathbf{i}^{d}=$ $[0,2]$, a nova classe de estados $C E_{1}=\left\langle\mathrm{M}_{1}, \mathbf{I}_{1}\right\rangle$ é dada por:

$$
\begin{aligned}
& \mathrm{M}_{1}=\left[\begin{array}{lllll}
1 & 0 & 1 & 0 & 0
\end{array}\right]^{T} \\
& \mathrm{M}_{1} \geq \operatorname{Pre}\left(t_{1}\right) \Rightarrow \mathrm{h}_{1}=\left[\begin{array}{llll}
1 & 0 & 0 & 0
\end{array}\right]^{T}
\end{aligned}
$$

$\mathrm{O}$ vetor $\mathrm{n}_{1}$ das transições recém-habilitadas é obtido pela diferença entre o vetor $h_{1}$ das transições habilitadas e o vetor $h_{0}$ subtraído da transição que disparou. Assim,

$$
\begin{aligned}
& \mathrm{n}_{1}=\mathrm{h}_{1}-\left(\mathrm{h}_{0}-\mathrm{q}\right)=0 \Rightarrow \mathrm{p}_{1}=\mathrm{h}_{1} \\
& \mathbf{I}_{1}=\mathbf{I}_{0} \mathrm{p}_{1}=\left(\begin{array}{llll}
{[1,2]} & 0 & 0 & 0
\end{array}\right)^{T}
\end{aligned}
$$

- Analogamente, disparando a transição $t_{1}$ com $q=$ $\left[\begin{array}{cccc}1 & 0 & 0 & 0\end{array}\right]^{T}$ no intervalo $\mathbf{i}^{d}=[1,2]$, uma nova classe de estados $C E_{2}=\left\langle\mathrm{M}_{2}, \mathbf{I}_{2}\right\rangle$ é alcançada com:

$$
\begin{aligned}
& \mathrm{M}_{2}=\left[\begin{array}{lllll}
0 & 0 & 1 & 1 & 0
\end{array}\right]^{T} \\
& \mathrm{M}_{2} \geq \operatorname{Pre}\left(t_{3}\right) \Rightarrow \mathrm{h}_{2}=\left[\begin{array}{llll}
0 & 0 & 1 & 0
\end{array}\right]^{T} \\
& \mathrm{n}_{2}=\mathrm{h}_{2}-\left(\mathrm{h}_{1}-\mathrm{q}\right)=\mathrm{h}_{2} \Rightarrow \mathrm{p}_{2}=0 \\
& \mathbf{I}_{1}=\left(\mathbf{i}^{d} \mathrm{u}+\mathbf{I}^{s}\right) \mathrm{n}_{2}=\left(\begin{array}{llll}
0 & 0 & {[1,2]} & 0
\end{array}\right)^{T}
\end{aligned}
$$

- Finalmente, disparando a transição $t_{3}$ com $q=$ $\left[\begin{array}{llll}0 & 0 & 1 & 0\end{array}\right]^{T}$ no intervalo $\mathbf{i}^{d}=[1,2]$, uma nova classe estados $C E_{3}=\left\langle\mathrm{M}_{3}, \mathbf{I}_{3}\right\rangle$ é alcançada com:

$$
\begin{aligned}
& \mathrm{M}_{3}=\left[\begin{array}{lllll}
0 & 0 & 0 & 0 & 1
\end{array}\right]^{T} \\
& \mathrm{M}_{3} \geq \operatorname{Pre}\left(t_{4}\right) \Rightarrow \mathrm{h}_{3}=\left[\begin{array}{llll}
0 & 0 & 0 & 1
\end{array}\right]^{T} \\
& \mathrm{n}_{3}=\mathrm{h}_{3}-\left(\mathrm{h}_{2}-\mathrm{q}\right)=\mathrm{h}_{3} \Rightarrow \mathrm{p}_{3}=0 \\
& \mathbf{I}_{3}=\left(\mathbf{i}^{d} \mathrm{u}+\mathbf{I}^{s}\right) \mathrm{n}_{3}=\left(\begin{array}{llll}
0 & 0 & 0 & {[1,4}
\end{array}\right)^{T}
\end{aligned}
$$

O intervalo $\mathbf{I}_{3}(4)=[1,4]$ é o intervalo de disparo da transição $t_{4}$ contado a partir do início da execução da rede. Qualquer que seja o caminho usado para, a partir da marcação $\mathrm{M}_{0}$, alcançar a marcação $\mathrm{M}_{3}$, o intervalo de disparo estará contido no intervalo $[1,4]$. Assim, o intervalo $\mathbf{I}_{3}$ é uma aproximação intervalar externa.

\section{CARACTERIZAÇÃO DOS DOMÍNIOS DE TEMPO EM UMA RPT}

Nos métodos de análise das RPTs encontrados na literatura, a caracterização dos domínios de tempo de uma classe alcançável pelo disparo de uma sequência de transições só é possível com a execução de algum algoritmo que explicite todas as classes alcançáveis por esta seqüência. Nesta seção é apresentado o problema da caracterização de domínios de tempo para uma classe de estados qualquer, conforme o enunciado a seguir:

Seja $C E_{0}$ uma classe de estados inicial de uma RPT. Assumindo que a seqüência de transições $\sigma_{i}=$ $t_{1}, t_{2} \ldots, t_{n}$ é executável a partir de $C E_{0}$ e que tal execução leva a rede à classe $C E_{n}$, como então caracterizar os domínios de tempo da classe $C E_{n}$ sem que seja necessário explicitar todas as classes intermediárias?

Este problema é ilustrado na Figura 4, na qual a classe $C E_{n}$ é alcançável a partir da classe inicial $C E_{0}$ através de diversos caminhos. Porém, deseja-se caracterizar $C E_{n}$ de modo que qualquer caminho que leve $C E_{0}$ a $C E_{n}$ esteja incluído nessa caracterização.

A solução para tal problema é obtida em Lima et al. (2006) se a $R P T$ pertencer a uma classe de redes denominadas nãopersistentes $^{1}$. Nesta classe, nenhuma transição permanece

\footnotetext{
${ }^{1}$ as redes não persistentes podem ser do tipo grafo marcado, máquina de estado ou livre escolha, por exemplo.
} 


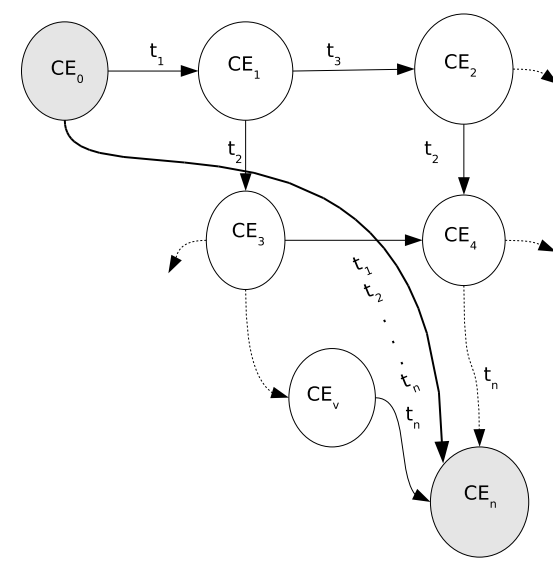

Figura 4: Grafo de classes, sendo $C E_{n}$ a classe final e $C E_{0}$ a classe inicial.

habilitada quando há uma mudança de classe. Logo, $\mathrm{p}=0$ (vetor nulo) e $\mathrm{h}=\mathrm{n}$ em qualquer classe de estado. Neste caso, a Equação 8 assume a seguinte forma:

$$
\mathbf{I}_{1}=\left(\mathbf{I}^{d} \mathrm{u}+\mathbf{I}^{s}\right) \mathrm{n}_{1}
$$

Aplicando-se a Equação 10 numa seqüência de transições $\sigma=t_{1} t_{2} \ldots t_{n}$ que leva a rede desde a classe $C E_{0}$ até $C E_{n}$, cada transição habilitada na classe $C E_{n}$ terá um domínio de tempo $\mathbf{i}_{n}$ dado por:

$$
\mathbf{i}_{n}=\sum_{i=1}^{n} \mathbf{I}^{s}\left(\sigma_{i}\right)
$$

A Equação 11 pode ser escrita como sendo o produto intervalar entre o vetor domínio estático da rede $\mathbf{I}^{s}$ e o vetor pontual $\mathrm{q}$, representando o vetor de disparo de uma sequiência de transições. Assim,

$$
\mathbf{i}_{n}=\left(\mathbf{I}^{s}\right)^{T} \mathrm{q}
$$

Considerando $\mathbf{c}=\left(\mathbf{I}^{S}\right)^{T}$, a Equação 12 assume a forma de uma equação linear intervalar do tipo

$$
\mathbf{i}=\mathbf{c} q
$$

sendo $\mathbf{c}^{T} \in \mathbb{R}^{n}, \mathbf{i} \in \mathbb{I}, \mathrm{q} \in\left(\mathbb{Z}^{+}\right)^{n}$ e $n$ o número de transições da rede.

A Equação 13 permite calcular os domínios de disparo em uma $R P T$ tanto pelo disparo transição a transição, quanto pelo disparo de uma sequiência de transições.

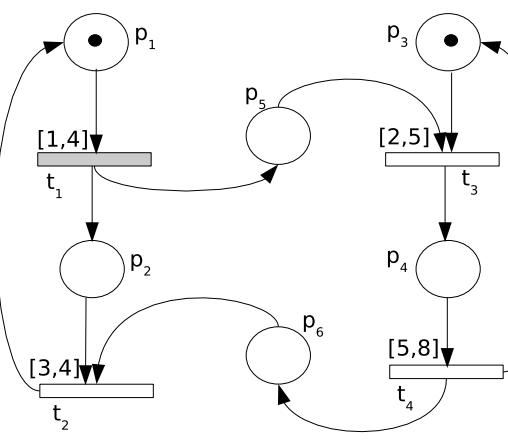

Figura 5: Exemplo de uma RPT closed-loop

Apesar da restrição $p=0$, a Equação 13 é um importante resultado para o uso de uma nova ferramenta matemática na análise de uma $R P T$. O intervalo i contém os limites mínimo e máximo de tempo para a execução de uma seqüência que, juntamente com a equação de estado das redes de Petri convencionais, mapeiam toda a evolução da rede.

Exemplo 5.1 A $R P T$ mostrada na Figura 5 é uma rede segura denominada closed-loop, muito utilizada na modelagem de protocolos de comunicação. Um transmissor envia uma mensagem a partir de uma caixa eletrônica e também ativa uma unidade de recepção de uma mensagem. Um receptor de mensagens, localizado no outro extremo do sistema, recebe a mensagem e envia uma confirmação do recebimento. Na Tabela 1, é mostrado o significado de cada lugar e transição da rede.

Tabela 1: Significado dos lugares e transições da Figura 5

\begin{tabular}{|c|l|}
\hline Lugar & Descrição \\
\hline$p_{1}$ & transmissor pronto para enviar uma mensagem \\
$p_{2}$ & transmissor em estado de recepção \\
$p_{3}$ & receptor pronto para receber mensagem \\
$p_{4}$ & mensagem recebida pelo receptor \\
$p_{5}$ & mensagem sendo transmitida \\
$p_{6}$ & mensagem confirmando sucesso na recepção \\
\hline$t_{1}$ & transmissor enviou mensagem \\
$t_{2}$ & transmissor elabora nova mensagem \\
$t_{3}$ & receptor recebeu mensagem \\
$t_{4}$ & receptor envia confirmação \\
\hline
\end{tabular}

Neste exemplo, tem-se:

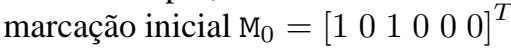

vetor domínio de tempo estático

$$
\mathbf{I}^{s}=([1,4][3,4][2,5][5,8])^{T}
$$

Substituindo-se estes valores na Equação 13 é tem-se: 


$$
\mathbf{i}=\left(\begin{array}{c}
{[1,4]} \\
{[3,4]} \\
{[2,5]} \\
{[5,8]}
\end{array}\right)^{T}\left(\begin{array}{l}
q_{1} \\
q_{2} \\
q_{3} \\
q_{4}
\end{array}\right)
$$

1. para $q=\left[\begin{array}{llll}1 & 1 & 1 & 1\end{array}\right]^{T}$, ou seja, disparando todas as transições da rede, o domínio de disparo da última transição é obtido como segue:

$$
\mathbf{i}=\left(\begin{array}{l}
{[1,4]} \\
{[3,4]} \\
{[2,5]} \\
{[5,8]}
\end{array}\right)^{T}\left(\begin{array}{l}
1 \\
1 \\
1 \\
1
\end{array}\right)=[11,21]
$$

O resultado obtido com a Equação 13 representa o intervalo de tempo para execução dessa seqüência.

Devido a $\mathrm{M}_{0}$, o vetor $q$ representa seqüência a seguir:

$$
\mathrm{M}_{0} \stackrel{t_{1}}{\longrightarrow} \mathrm{M}_{1} \stackrel{t_{3}}{\longrightarrow} \mathrm{M}_{2} \stackrel{t_{4}}{\longrightarrow} \mathrm{M}_{3} \stackrel{t_{2}}{\longrightarrow} \mathrm{M}_{0}^{\prime}
$$

2. Cada transição da rede tem seu domínio de tempo global de disparo e esse valor depende do instante de ocorrência na sequiência. Assim,

- para o primeiro ciclo, os tempos globais das transições são calculados como segue:

$$
\begin{aligned}
\mathbf{I}_{G}^{1}\left(t_{1}\right) & =[1,4] \\
\mathbf{I}_{G}^{1}\left(t_{3}\right) & =\mathbf{I}_{G}^{1}\left(t_{1}\right)+\mathbf{I}^{s}\left(t_{3}\right)=[3,9] \\
\mathbf{I}_{G}^{1}\left(t_{4}\right) & =\mathbf{I}_{s}\left(t_{4}\right)+\mathbf{I}_{G}^{1}\left(t_{3}\right)=[8,17] \\
\mathbf{I}_{G}^{1}\left(t_{2}\right) & =\mathbf{I}_{s}\left(t_{2}\right)+\mathbf{I}_{G}^{1}\left(t_{4}\right)=[11,21]
\end{aligned}
$$

- para os demais ciclos, o tempo global de cada transição é obtido adicionando o tempo do primeiro ciclo ao tempo de ciclo da rede vezes o número de ciclos ocorridos, ou seja,

$$
\mathbf{I}_{G}^{k+1}=\mathbf{I}_{G}^{1}+\mathbf{d} k u,
$$

sendo $\mathbf{I}_{G}^{k+1}$ o vetor dos intervalos de tempo global após $k+1$ disparos de cada transição; $\mathbf{d}=$ $[\underline{d}, \bar{d}] \in \mathbb{I} \mathbb{R}$ é o intervalo de tempo de um ciclo.

- Na Tabela 2 são mostrados os tempos globais para os primeiros três ciclos da rede.

Assim, a Equação 13 permite obter não só a mudança de uma classe de estados para uma outra qualquer, mas também pode ser usada para verificar datas de disparos e calcular o tempo entre duas ocorrências quaisquer.

Na seção seguinte é apresentado um método de redução que possibilita ampliar o escopo das RPTs em que a Equação 13 é aplicável.
Tabela 2: Tempos globais de disparo para cada transição

\begin{tabular}{c|c|c|c|c}
\hline$\sigma$ & $\mathbf{I}^{s}$ & $\begin{array}{c}\mathbf{I}_{G}^{1} \\
k=0\end{array}$ & $\begin{array}{c}\mathbf{I}_{G}^{2} \\
k=1\end{array}$ & $\begin{array}{c}\mathbf{I}_{G}^{3} \\
k=2\end{array}$ \\
\hline$t_{1}$ & {$[1,4]$} & {$[1,4]$} & {$[12,25]$} & {$[23,46]$} \\
\hline$t_{3}$ & {$[2,5]$} & {$[3,9]$} & {$[14,30]$} & {$[25,51]$} \\
\hline$t_{4}$ & {$[5,8]$} & {$[8,17]$} & {$[19,38]$} & {$[40,59]$} \\
\hline$t_{2}$ & {$[3,4]$} & {$[11,21]$} & {$[22,42]$} & {$[33,63]$} \\
\hline
\end{tabular}

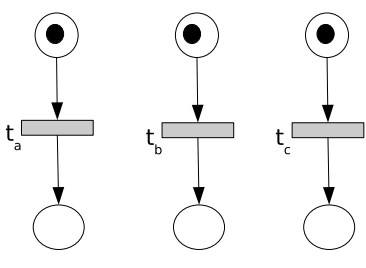

(a)

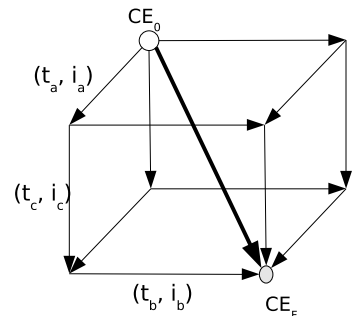

(b)
Figura 6: (a) Transições concorrentes (b) Espaço de estado completo e reduzido

\section{REDUÇÃO DE UMA REDE DE PETRI TEMPORAL}

Em Lima (2007) é apresentado um conjunto de regras para redução de RPTs através do conceito de aproximação intervalar. A rede mostrada na Figura 6 ilustra a idéia básica da redução, onde as transições $t_{a}, t_{b}$ e $t_{c}$ são chamadas de independentes, pois não existe qualquer relação entre suas habilitações e disparos. Na classe de estados inicial $C E_{0}$, as três transições estão habilitadas. Uma classe de estados final $C E_{F}$ é alcançável pelo disparo das três transições, independente da ordem de ocorrência. Dessa forma, a redução visa obter uma rede equivalente que caracterize o domínio de tempo da classe final para algumas transições de interesse.

Sejam $t_{1}$ e $t_{2}$, duas transições de uma RPT. Os domínios estáticos de $t_{1}$ e $t_{2}$ são $\mathbf{i}^{s}\left(t_{1}\right)=\left[\delta_{1}, \Delta_{1}\right]$ e $\mathbf{i}^{s}\left(t_{2}\right)=\left[\delta_{2}, \Delta_{2}\right]$, respectivamente e a condição $\mathbf{i}^{s}\left(t_{1}\right) \cap \mathbf{i}^{s}\left(t_{2}\right) \neq \emptyset$ deve ser satisfeita. As regras de redução são apresentadas abaixo.

1. As transições $t_{1}$ e $t_{2}$ são ditas concorrentes $\left(t_{1} \| t_{2}\right)$ se existirem os lugares $p_{1}$ e $p_{2}$, tais que:

(a) $p_{1}$ e $p_{2}$ têm a mesma transição de entrada, ou seja, ${ }^{\bullet} p_{1}={ }^{\bullet} p_{2}=\left\{t_{0}\right\}$.

(b) $p_{1}$ e $p_{2}$ têm uma única transição de saída, ou seja, $p_{1}^{\bullet}=\left\{t_{1}\right\}$ e $p_{2}^{\bullet}=\left\{t_{2}\right\}$. 


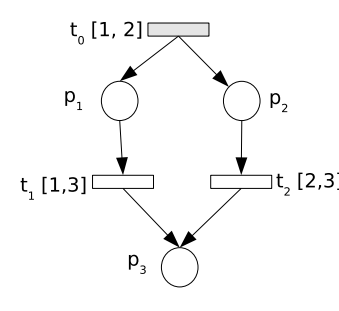

(a)

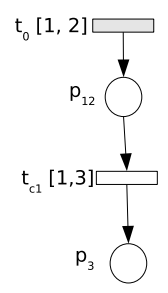

(b)
Figura 7: Redução de transições concorrentes

Aproximação Intervalar:

As transições $t_{1}$ e $t_{2}$ podem ser substituídas por uma transição $t_{p}$, com intervalo estático igual à união intervalar dos intervalos estáticos das transições $t_{1}$ e $t_{2}$,

$$
\mathbf{i}\left(t_{p}\right)=\left[\min \left\{\delta_{1}, \delta_{2}\right\}, \max \left\{\Delta_{1}, \Delta_{2}\right\}\right] .
$$

Na Figura 7 é mostrada a junção das trasições $t_{1}$ e $t_{2}$.

2. As transições $t_{1}$ e $t_{2}$ mostradas na Figura 8 , estão em série, pois ambas ou nenhuma delas podem ser disparadas. De forma geral, para que essa condição exista é preciso que $t_{1}$ e $t_{2}$ estejam separadas por um lugar $p$, tal que:

(a) O lugar $p$ não contém marca inicial, $M_{0}(p)=0$.

(b) O lugar p é o único lugar de saída de $t_{1}$ e o único lugar de entrada de $t_{2}$, ou seja, $t_{1}^{\bullet}={ }^{\bullet} t_{2}=\{p\}$.

(c) O lugar p está conectado apenas às transições $t_{1}$ e $t_{2}$, de modo que ${ }^{\bullet}=t_{1}$ e $p^{\bullet}=t_{2}$.

(d) $\forall p_{0} \in \bullet^{\bullet} t_{1}$ tem-se $p_{0}^{\bullet}=\left\{t_{1}\right\}$, exceto se $\boldsymbol{i}^{s}\left(t_{2}\right)=$ $[0,0]$.

Aproximação Intervalar:

Neste caso, $t_{1}$ e $t_{2}$ podem ser trocadas por uma transição $t_{s}$, com intervalo estático calculado como segue:

$$
\mathbf{i}^{s}\left(t_{s}\right)=\mathbf{i}^{s}\left(t_{1}\right)+\mathbf{i}^{s}\left(t_{2}\right)
$$

3. As transições $t_{1}$ e $t_{2}$ são conflitantes se as seguintes condições forem satisfeitas:

(a) $t_{1}$ e $t_{2}$ têm o mesmo lugar de entrada, ou seja, $\exists p_{0}$ tal que $\boldsymbol{t}_{1}=\boldsymbol{\bullet}_{2}=\left\{p_{0}\right\}$.

(b) O lugar $p_{0}$ é seguro e tem, como conjunto das transições de saída, as transições conflitantes, ou seja, $p_{0}^{\bullet}=\left\{t_{1}, t_{2}\right\}$

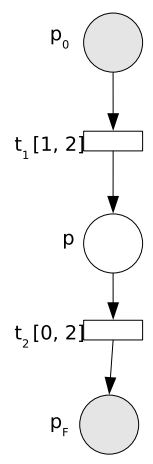

(a)

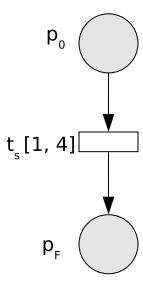

Figura 8: Redução das transições em série $t_{1}$ e $t_{2}$

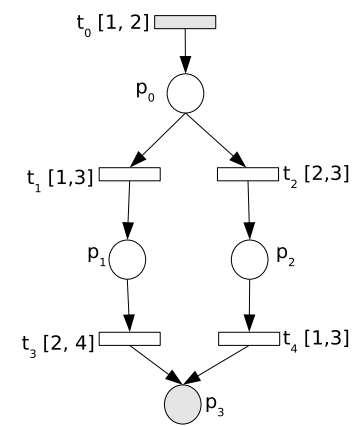

(a)

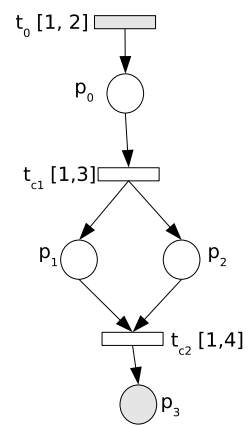

(b)
Figura 9: Redução das transições conflitantes $t_{1}$ e $t_{2}\left(t_{3}\right.$ e $t_{4}$ concorrentes)

Aproximação Intervalar:

$\mathrm{O}$ intervalo da transição equivalente $t_{c}$, mostrado na Figura 9, é calculado como segue:

$$
\mathbf{i}\left(t_{c_{1}}\right)=\left[\min \left\{\delta_{1}, \delta_{2}\right\}, \min \left\{\Delta_{1}, \Delta_{2}\right\}\right] .
$$

Note que a junção das transições $t_{1}$ e $t_{2}$ levou à concorrência das transições $t_{3}$ e $t_{4}$, cuja junção é feita usando a Equação 15 . Neste caso, ocorre um alargamento do intervalo equivalente. Porém, o intervalo da rede original estará contido neste intervalo, seja qual for o caminho.

As regras de redução apresentadas nesta seção têm por objetivo obter uma rede reduzida sem a presença de transições persistentes. Esta redução pode ser realizada para avaliar o comportamento de transições específicas que representem, por exemplo, tarefas críticas de um sistema. Eventualmente, a redução pode ampliar os 
intervalos originais, mas garante que estes intervalos estarão incluídos nos intervalos da rede reduzida. Isso possibilitará estimar o intervalo de realização de uma seqüência de disparo de transições sem que seja necessário executar a rede, permitindo ainda generalizar os resultados da Seção 5, conforme apresentado a seguir.

\section{EQUAÇÃO INTERVALAR GERAL PARA UMA RPT}

A partir dos resultados da Seção 6, é possível ampliar a classe das RPTs para as quais a Equação 13 pode ser aplicada. Uma $R P T$ passível de ser reduzida à forma de uma rede não persistente pode ser representada por uma matriz intervalar C como segue:

$$
\mathbf{C}=\left\{\begin{array}{lll}
\mathbf{C}(i, i)=\mathbf{I}^{s}(i), & \\
\mathbf{C}(i, j)=\mathbf{I}^{s}(j), & j \leq i & \text { se } t_{i} \text { precede } t_{j} \\
\mathbf{C}(i, j)=0, & j \leq i & \text { se } t_{i} \text { não precede } t_{j} \\
\mathbf{C}(i, j)=0, & j>i &
\end{array}\right.
$$

sendo $i, j=1, \ldots, n$ e $n$ o número de transições da rede reduzida. Uma transição $t_{i}$ precede uma transição $t_{j}$ se existe um caminho de $t_{i}$ a $t_{j}$.

Exemplo 7.1 Na Figura 10 é mostrada uma rede tipo grafo marcado e sua equivalente reduzida à forma não persistente, usando as regras da seção anterior.

As transições $t_{2}$ e $t_{3}$ da Figura 10a, cujos intervalos são $[1,3]$ e $[2,3]$, respectivamente, podem ser agrupadas formando uma nova transição com intervalo $[1,3]$ na Figura $10 b$, conforme Equação 15. Este procedimento é repetido com as transições $t_{4}$ e $t_{5}$ da Figura 10a. Finalmente, obtém-se a matriz $\mathbf{C}$ para a rede reduzida da Figura 10 b como

$$
\mathbf{C}=\left(\begin{array}{cccc}
{[1,2]} & 0 & 0 & 0 \\
{[1,2]} & {[1,3]} & 0 & 0 \\
{[1,2]} & {[1,3]} & {[1,4]} & 0 \\
{[1,2]} & {[1,3]} & {[1,4]} & {[1,2]}
\end{array}\right)
$$

Utilizando a matriz $C$, pode-se escrever a Equação 13 na forma matricial como

$$
\mathbf{I}=\mathbf{C} q_{d}
$$

sendo $\mathbf{C} \in \mathbb{I} \mathbb{R}^{r \times r}$ a matriz de intervalos da $R P T$ reduzida, $q_{d} \in\left(\mathbb{Z}^{+}\right)^{r}$ o vetor quantidade de disparos das transições e $\mathbf{I} \in \mathbb{I R}^{r}$ o vetor domínio dinâmico da rede reduzida.

A matriz C da Equação 19 representa a relação de precedência entre as transições. Neste caso, a Equação 13

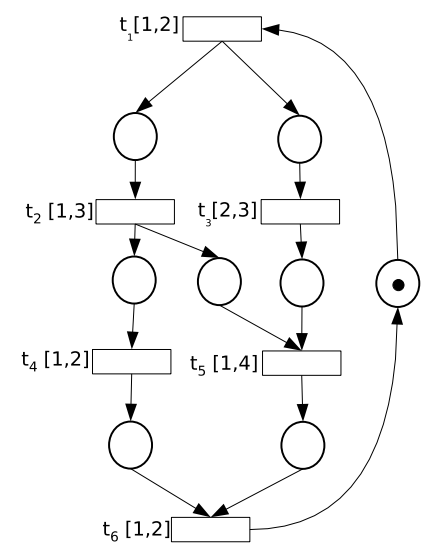

(a)

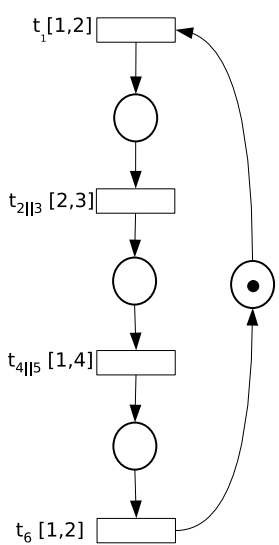

(b)
Figura 10: Redução de uma RPT: (a) original e (b) reduzida

passa a ser um caso particular da Equação 19, pois c é a última linha da matriz C. Esta linha contém as relações de precedência da transição que depende de todas as outras.

Neste caso, a Equação 9 adquire a forma

$$
\left\{\begin{array}{l}
\mathrm{M}=\mathrm{M}_{0}+\mathrm{Dq}_{d} \\
\mathbf{I}=\mathbf{C q}_{d}
\end{array}\right.
$$

sendo $\mathrm{D}=$ Pos - Pre a matriz de incidência da rede.

Considerando a rede mostrada na Figura 10b, o intervalo de tempo de disparo para um ciclo de execução da seqüência de disparo $t_{1}-t_{2}-t_{3}-t_{4}$, ou seja, para $q_{d}=\left[\begin{array}{llll}1 & 1 & 1 & 1\end{array}\right]^{T}$ é dado por:

$$
\mathbf{I}=\mathbf{C} q_{d}=\left(\begin{array}{c}
{[1,2]} \\
{[2,5]} \\
{[4,9]} \\
{[5,11]}
\end{array}\right)
$$

Portanto, para uma rede cíclica segura, o cálculo do domínio do tempo para o disparo de uma sequiência de transições é obtido diretamente da soma dos intervalos que compõem a rede equivalente.

O vetor I é, portanto, uma aproximação intervalar contendo desde o menor até o maior tempo para execução de uma determinada seqüência de disparo.

Para avaliar o tempo global de cada transição pertencente à seqüência, aplica-se a Equação 14. Para o primeiro ciclo de execução da rede, cada elemento do vetor I da Equação 20 representa o tempo global de cada transição. Assim,

$$
\mathbf{I}_{G}^{1}=\mathbf{I}
$$

Para o segundo ciclo em diante, 


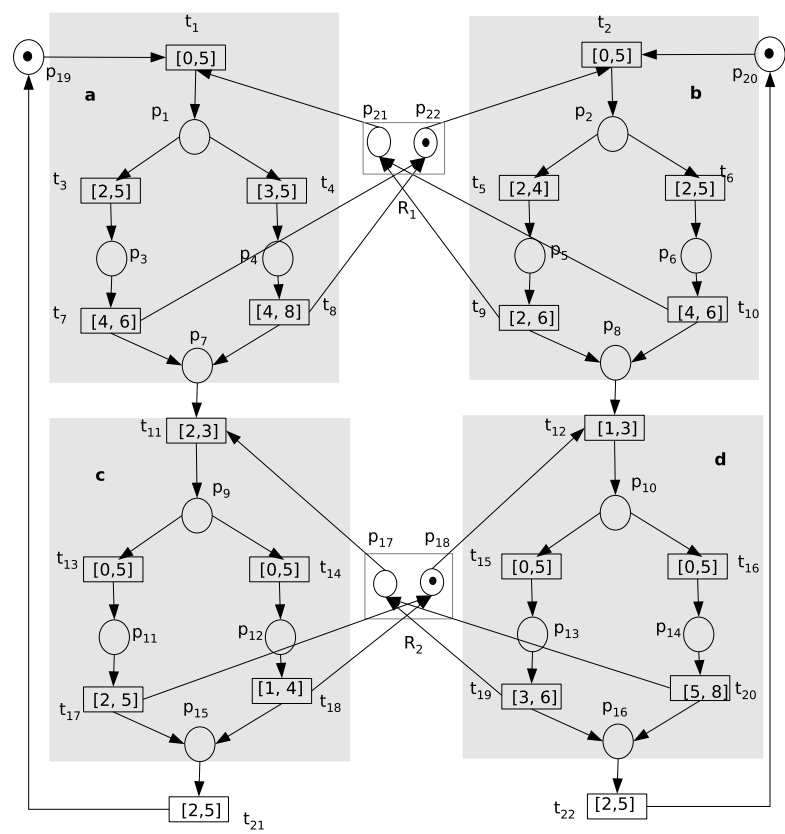

Figura 11: $R P T$ de um escalonador de tarefas

$$
\begin{gathered}
\mathbf{I}_{G}^{2}=\mathbf{I}+\mathbf{d} \mathrm{u}=\left(\begin{array}{c}
{[1,2]} \\
{[3,5]} \\
{[4,9]} \\
{[5,11]}
\end{array}\right)+[5,11]\left(\begin{array}{l}
1 \\
1 \\
1 \\
1
\end{array}\right) \\
\mathbf{I}_{G}^{2}=\left(\begin{array}{c}
{[6,13]} \\
{[8,16]} \\
{[9,20]} \\
{[10,22]}
\end{array}\right)
\end{gathered}
$$

sendo $\mathbf{d}=[5,11]$ o ciclo de execução da rede obtido da Equação 21.

\section{APLICAÇÃO}

Na Figura 11 é mostrado o modelo em RPT de um sistema temporizado que representa, por exemplo, um sistema de manufatura onde recursos são utilizados para executar diferentes tarefas. Os recursos $\mathbf{R}_{1}$ e $\mathbf{R}_{2}$ são utilizados para execução de quatro diferentes tarefas: $\mathbf{a}, \mathbf{b}, \mathbf{c}$, e d. No estado inicial o recurso $\mathbf{R}_{1}$ está disponível para a tarefa $\mathbf{b}$, que tem prioridade sobre as demais tarefas. Ao término da tarefa $\mathbf{b}$, o recurso $\mathbf{R}_{1}$ será utilizado pela tarefa $\mathbf{a}$, enquanto o recurso $\mathbf{R}_{2}$ estará disponível para execução da tarefa $\mathbf{d}$, a qual tem prioridade sobre a tarefa $\mathbf{c}$. A tarefa $\mathbf{c}$ deve utilizar o recurso $\mathbf{R}_{2}$. Na Tabela 3 são descritos os significados dos lugares e transições da rede.

Aplicando-se o método de redução visto na Seção 6, obtém-
Tabela 3: Significado dos lugares e transições da $R P T$ da Figura 11

\begin{tabular}{|c|l|}
\hline Lugar & Descrição \\
\hline$p_{1}, p_{2}, p_{9}, p_{10}$ & lugares de decisão \\
$p_{3}, \ldots, p_{6} \mathrm{e} p_{11}, \ldots, p_{14}$ & execuções \\
$p_{7}, p_{8}, p_{15}, p_{16}$ & fim das tarefas \\
$p_{19}, p_{20}$ & sistema livre \\
$p_{17}, p_{18}, p_{21}, p_{22}$ & recursos livres \\
\hline$t_{1}, t_{2}, t_{11}, t_{12}$ & solicita recurso \\
$t_{3}, \ldots, t_{10} \mathrm{e} t_{13}, \ldots, t_{20}$ & início e fim das tarefas \\
$t_{21}, t_{22}$ & reinício do ciclo \\
\hline
\end{tabular}

se as redes reduzidas mostradas nas Figuras 12 e 13.

A

Figura 12: $R P T$ reduzida representando as tarefas

$t_{b}$

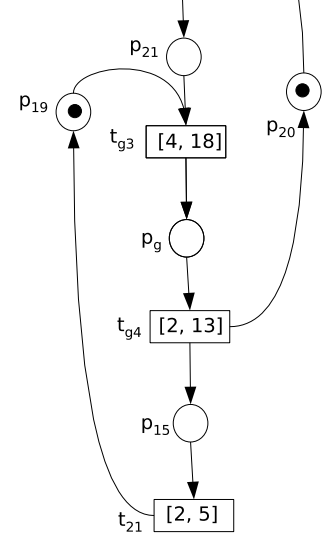

Figura 13: $R P T$ reduzida não persistente 
rede mostrada na Figura 12 representa uma rede reduzida a partir da rede original, substituindo cada tarefa por uma transição equivalente ao intervalo de tempo para realização da tarefa. A partir da rede mostrada na Figura 12, obtém-se a rede equivalente reduzida mostrada na Figura 13, a qual é uma rede não persistente.

A partir da rede mostrada na Figura 13 e da Equação 18, obtém-se a seguinte matriz intervalar:

$$
\mathbf{C}=\left(\begin{array}{cccc}
{[4,15]} & 0 & 0 & 0 \\
{[4,15]} & {[4,18]} & 0 & 0 \\
{[4,15]} & {[4,18]} & {[2,13]} & 0 \\
{[4,15]} & {[4,18]} & {[2,13]} & {[2,5]}
\end{array}\right)
$$

Substituindo-se a matriz C na Equação 19, é possível caracterizar classes de estados, tempos para execução de determinadas tarefas do sistema, além de algumas medidas de desempenho do sistema sem executar a rede.

\section{Intervalo de tempo para um ciclo}

Um ciclo representa o tempo, ou melhor, um intervalo de tempo para se completar um serviço, correspondente ao tempo entre a habilitação da transição $t_{b}$ e o disparo da transição $t_{21}$. Usando a equação intervalar para o sistema, obtém-se o intervalo de tempo como segue:

$$
\mathbf{I}=\mathbf{C} q_{d}=\left(\begin{array}{c}
{[4,15]} \\
{[8,33]} \\
{[10,46]} \\
{[12,51]}
\end{array}\right)
$$

sendo $q_{d}=\left[\begin{array}{llll}1 & 1 & 1 & 1\end{array}\right]^{T}$.

$\mathrm{O}$ intervalo $[12,51]$ caracteriza o intervalo para os possíveis tempos necessários para se completar um serviço e esses tempos dependem do instante de disparo inicial. Por exemplo, para um disparo de $t_{b}$ no instante 10 unidades de tempo, o intervalo para completar um ciclo é igual a $[18,46]$.

\section{Tempo global para execução de cada tarefa}

$\mathrm{Na}$ rede reduzida, os tempos estão associados às transições agrupadas e correspondem à execução conjunta de tarefas. Os tempos globais dessas tarefas podem ser obtidos executando-se a rede original ou a partir dos tempos da rede reduzida equivalente, como mostrado adiante.

Para a rede reduzida mostrada na Figura 13, as transições $t_{g 3}$ e $t_{g 4}$ fornecem os seguintes tempos globais:

$\mathbf{i}_{G}\left(t_{g 3}\right)=[8,33]$

$\mathbf{i}_{G}\left(t_{g 4}\right)=[10,46]$
A transição $t_{g 3}$ resulta do agrupamento $t_{g 3}=t_{a} \| t_{d}$. Logo,

$\mathbf{i}\left(t_{g 3}\right)=\left[\min \left\{\delta_{a}, \delta d\right\}, \max \left\{\Delta_{a}, \Delta d\right\}\right]$.

Sendo $\mathbf{i}\left(t_{a}\right)=[6,18]$ e $\mathbf{i}\left(t_{d}\right)=[4,16]$ tem-se :

$$
\begin{gathered}
t_{g 3}:[4,18] \stackrel{\mathbf{d}}{\longrightarrow}[8,33] \\
t_{a}:[6,18] \stackrel{\mathbf{d}}{\longrightarrow}[?, 33] \\
t_{d}:[4,16] \stackrel{\mathbf{d}}{\longrightarrow}[8, ?]
\end{gathered}
$$

Como $\mathbf{i}_{G}\left(t_{g 3}\right)$ e $\mathbf{i}\left(t_{g 3}\right)$ são intervalos dependentes, $\mathbf{d}$ pode ser calculado usando a subtração dependente como segue:

$\mathbf{i}_{G}\left(t_{g 3}\right)=\mathbf{i}\left(t_{g 3}\right)+\mathbf{d}$

$\mathbf{d}=\mathbf{i}_{G}\left(t_{g 3}\right) \ominus \mathbf{i}\left(t_{g 3}\right)=[4,15]$

Assim,

$$
\begin{gathered}
\mathbf{i}_{G}\left(t_{a}\right)=\mathbf{i}\left(t_{a}\right)+d=[10,33] \\
\mathbf{i}_{G}\left(t_{d}\right)=\mathbf{i}\left(t_{d}\right)+d=[8,31]
\end{gathered}
$$

Repetindo o mesmo procedimento para a transição $t_{g 4}$, tem-se:

$$
\begin{aligned}
& t_{g 4}:[2,13] \stackrel{\mathbf{d}}{\longrightarrow}[10,46] \\
& t_{c}:[3,13] \stackrel{\mathbf{d}}{\longrightarrow}[?, 46] \\
& t_{22}:[2,5] \stackrel{\mathbf{d}}{\longrightarrow}[10, ?]
\end{aligned}
$$

Como $\mathbf{d}=\mathbf{i}_{G}\left(t_{g 4}\right) \ominus \mathbf{i}\left(t_{g 4}\right)=[8,33]$, os tempos globais das transições $t_{c}$ e $t_{22}$ são:

$$
\begin{aligned}
\mathbf{i}_{G}\left(t_{c}\right) & =[3+8,46]=[11,46] \\
\mathbf{i}_{G}\left(t_{22}\right) & =[10,5+33]=[10,38]
\end{aligned}
$$

A Tabela 4 mostra os tempos globais e relativos para cada tarefa.

Tabela 4: Tempos globais e relativos de cada tarefa

\begin{tabular}{c|c|c}
\hline Tarefa & Tempo Global & Tempo Relativo \\
\hline $\mathbf{b}$ & {$[4,15]$} & {$[4,15]$} \\
\hline $\mathbf{a}$ & {$[10,33]$} & {$[6,18]$} \\
\hline $\mathbf{d}$ & {$[8,31]$} & {$[4,16]$} \\
\hline $\mathbf{c}$ & {$[11,46]$} & {$[3,13]$} \\
\hline $\mathbf{2 2}$ & {$[10,38]$} & {$[2,7]$} \\
\hline $\mathbf{2 1}$ & {$[12,51]$} & {$[1,5]$} \\
\hline
\end{tabular}

Os intervalos das transições acima representam os tempos para execução de cada tarefa. A execução da tarefa $t_{a}$, por exemplo, corresponde à ocorrência da sequiência $t_{1}-t_{3}-t_{7}$ ou $t_{1}-t_{4}-t_{8}$.

Dessa forma, os intervalos de disparo das transições para classes de estados intermediários podem ser 


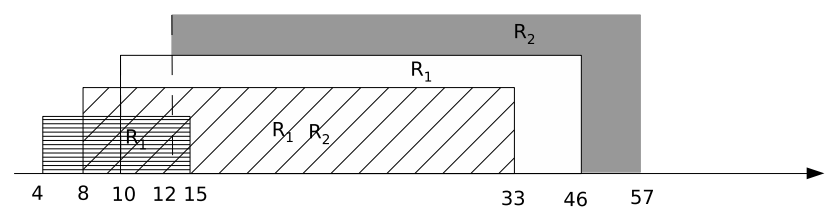

(a)

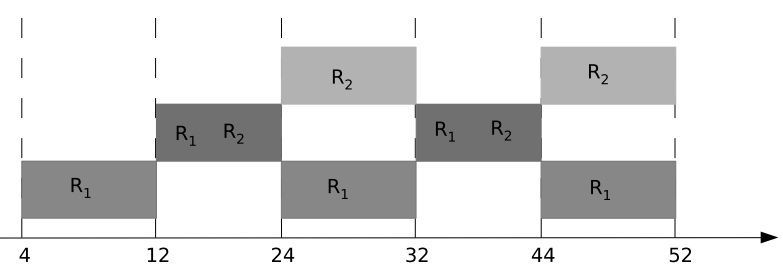

(b)

Figura 14: Utilização dos recursos $\mathbf{R}_{1}$ e $\mathbf{R}_{2}$

obtidos sem executar a rede e sem mapear o espaço de classes de estados completo. Por exemplo, o disparo da seqüência $t_{a}=t_{1}-t_{3}-t_{7}$ ou $t_{a}=t_{1}-t_{4}-t_{8}$ ocorre no intervalo de tempo global $[10,33]$. Este valor é contado a partir do estado inicial e considera o tempo global. Portanto, o intervalo de tempo da tarefa $t_{b}$ está contido neste intervalo. O tempo de execução da tarefa $t_{a}$ é obtido pela subtração dependente entre os intervalos de $t_{a}$ e $t_{b}$ como segue:

$$
\mathbf{i}\left(t_{a}\right)=\mathbf{i}_{G}\left(t_{a}\right) \ominus \mathbf{i}_{G}\left(t_{b}\right)=[6,18] .
$$

Esse tempo corresponde ao tempo relativo da tarefa $\mathbf{a}$, ou seja, seu tempo de execução.

\section{Utilização dos recursos}

A equação intervalar pode também ser aplicada para avaliação do uso dos recursos $\mathbf{R}_{1}$ e $\mathbf{R}_{2}$. Na Figura 14a, são mostrados os intervalos para possíveis execuções da rede reduzida mostrada na Figura 12. Nesta figura, observa-se uma ampla faixa de sobreposição nos tempos de utilização dos recursos. Porém, tais recursos são utilizados para execução de diferentes tarefas, sendo que um recurso não pode ser utilizado por mais de uma tarefa ao mesmo tempo. A partir das informações de operação do sistema e das mostradas na Figura 14a, pode-se determinar um escalonamento adequado para execução de cada tarefa, como mostrado na Figura 14b.

Para a utilização dos recursos sugerida na Figura 14b, os intervalos de tempo globais são calculados como segue:

$$
\mathbf{i}_{G}^{k}=\left(\begin{array}{c}
{[4,12]} \\
{[12,24]} \\
{[24,32]} \\
{[32,44]}
\end{array}\right)+20 k \mathbf{u}
$$

sendo $k$ o número de serviços completados e $\mathbf{d}=$ $[20,20]$ o tempo de ciclo.

\section{Redução do espaço de classes de estados}

A análise via alcançabilidade para a rede mostrada na Figura 11 torna-se inviável devido ao número elevado de classes de estados. Porém, considerando a rede mostrada na Figura 12, pode-se avaliar via alcançabilidade questões como: intervalos de tempo com variação da seqüência de execução das tarefas, bloqueio no uso dos recursos e tempo de espera. $\mathrm{Na}$ Figura 15, é mostrado parte do grafo de classes de estados para a rede reduzida da Figura 12.

Entretanto, devido à natureza do tempo global, o grafo de classes pode ser ilimitado. Uma forma de garantir a limitação do grafo de classes seria manter a rede com mesma seqüência. Com isso, o intervalo de tempo para um ciclo seria o mesmo e as classes com a mesma marcação teriam tempos proporcionais. Por exemplo, para a rede mostrada na Figura 15, sendo mantida a sequiência $\sigma=t_{b}-t_{a}-t_{d}-t_{c}-t_{22}-t_{21}$, o intervalo de tempo global para o disparo de cada transição é calculado conforme a Equação 14, ou seja:

$$
\mathbf{i}_{G}=\left(\begin{array}{c}
{[4,15]} \\
{[8,31]} \\
{[8,31]} \\
{[11,36]} \\
{[11,36]} \\
{[12,36]}
\end{array}\right)+[12,36] k \mathbf{u}
$$

Caso se amplie o intervalo de ciclo, novas seqüências poderão ser realizadas e mesmo assim se obter classes equivalentes, como é o caso das sequiências ressaltadas na Figura 15, em que o tempo de ciclo é ampliado para $[12,41]$. Com isso, a rede passa a ter 4 caminhos possíveis de serem seguidos, mantendo-se o tempo de ciclo dentro do estabelecido.

\section{CONCLUSÕES}

O problema de caracterização de intervalos de tempo de disparo de transições em RPTs foi apresentado e uma abordagem intervalar para análise deste problema foi proposta neste artigo. Enquanto as abordagens tradicionais, encontradas na literatura, não possibilitam a caracterização de uma classe de estados alcançável a partir de uma classe inicial pelo disparo de uma sequência de transições, na abordagem proposta isto é possível sem a necessidade da execução de um algoritmo que explicite todas as classes de estados alcançáveis. Utilizando recursos da álgebra intervalar, o problema da caracterização de intervalos de tempo de disparo em RPTs foi transformado em um 


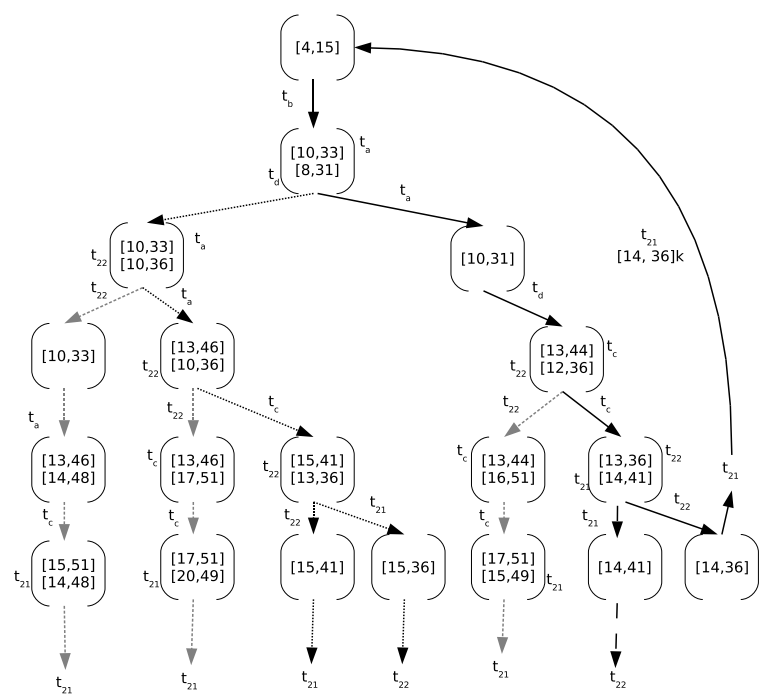

Figura 15: Espaço reduzido de classes de estados

problema de análise intervalar. Apesar da complexidade das redes temporais não seguras, por causa do forte efeito de intercalação dos disparos, a equação intervalar possibilita a estimação de algumas medidas quantitativas nestas redes. Dentre estas medidas tem-se: tempo de ciclo em redes reversíveis, taxa de disparo em redes acíclicas, tempo de execução de tarefas e tempo entre disparos de transições. Além disso, a equação possibilita a validação de seqüências de disparo de transições, o que permite, por exemplo, o planejamento de um escalonamento para execução de tarefas.

A partir da análise intervalar, também foi possível obterse outros resultados tais como a redução do espaço de classes de estados de uma RPT (delimitando um espaço de busca) e a definição de um conjunto de regras para redução de uma $R P T$, transformando a rede original em uma rede reduzida que preserva as restrições temporais e a viabilidade de ocorrência das transições.

\section{REFERÊNCIAS}

Alur, R. and Dill, D. L. (1994). A theory of timed automata, Theoretical Computer Science (126): 183-235.

Aura, T. and Lilius, J. (1996). Time processes for time Petri nets, Lecture Notes in Computer Science, Vol. 1248, Springer Verlag, Berlin, Germany, pp. 136-155.

Berthomieu, B. and Diaz, M. (1991). Modeling and verification of time dependent systems using Petri nets, IEEE Transactions on Software Engineering, Vol. 17, pp. 259-273.
Berthomieu, B. and Menasche, M. (1982). A state enumeration approach for analyzing time Petri nets, 3rd European Workshop on Applications and Theory of Petri Nets, Varenna, Italy.

Bucci, G. and Vicario, E. (1995). Compositional validation of time-critical systems using communicating time Petri nets, IEEE Transactions on Software Engeneering 21(12): 969-992.

Cerone, A. and Maggiolo-Schettini, A. (1999). Time-base expressivity of time Petri nets, Vol. 216, Theorical Computer Science, pp. 1-53.

Cohen, G. (2001). Analisis y Control de Sistemas de Eventos Discretos: De Redes de Petri Temporizadas al Algebra, Cuadernos del Instituto de Matematica Beppo Levi.

Gardey, G., Roux, O. H. and Roux, O. F. (2005). State space computation and analysis of time Petri nets, Theory and Practice of Logic Programming (TPLP). Special Issue on Specification Analysis and Verification of Reactive Systems.

Hansen, E. and Walster, G. W. (2004). Global Optimization Using Interval Analysis, Marcel Dekker.

Jaulin, L., Boimond, J. and Hardouin, L. (1999). Estimation of discrete-event systems using interval computation, Reliable Computing 5(2): 165-173.

Jaulin, L., Kieffer, M., Didrit, O. and Walter, E. (1995). Methods and Applications of Interval Analysis, SIAM Studies in Applied Mathematics.

Lilius, J. (1998). Efficient state space search for time Petri nets, Vol. 18, In Proc. MFCS'98 Workshop on Concurency.

Lima, E. A. (2007). Análise e Aplicações em Redes de Petri Temporais: Uma Abordagem via Álgebra Intervalar, $\mathrm{PhD}$ thesis, CPGEI - Universidade Tecnológica Federal do Paraná.

Lima, E. A., Lüders, R. and Künzle, L. A. (2005). Análise de redes de Petri temporais usando tempo global, VII Simpósio Brasileiro de Automação Inteligente - SBAI.

Lima, E. A., Lüders, R. and Künzle, L. A. (2006). Interval analysis of time Petri nets, 4th CESA Multiconference on Computational Engineering in Systems Applications.

Merlin, P. (1974). A Study of Recoverability of Computer Systems, PhD thesis, University of California IRVINE. available from Ann Arbor: Univ Microfilms, No. 7511026. 
Montano, L., Garci, F. J. and Villarroel, J. L. (2000). Using time Petri net formalism for specification, validation, and code generation in robot-control applications, The International Journal of Robotics Research 19(1): 5976.

Moore, R. E. (1995). Methods and Applications of Interval Analysis, SIAM - Studies in Applied Mathematics.

Murata, T. (1989). Petri nets: Properties, analysis and applications, Vol. 77, Proceedings of IEEE, pp. 541580.

Popova-Zeugmann, L. (1998). On time Petri nets, EIK: Journal of Information Processing and Cybernetics, Vol. 27, pp. 227-244.

Ramchandani, R. (1974). Analysis of Asyncronous Concurent Systems by Time Petri Nets, PhD thesis, Cambridge, Mass.: MIT, Dept. Electrical Engineering. Project MAC TR-120.

Tsai, J. J. P., Yang, S. J. and Chang, Y. H. (1995). Timming constraint Petri nets and their application to schedulability analsis of real-time systems specifications, IEEE Transactions on Software Engeneering 21(1): 32-49.

Vicario, E. (2001). Static analysis and dynamic steering of time-dependent systems, IEEE Transactions on Software Engeneering 27(8): 728-748.

Wang, J. and Deng, Y. (2000). Reachability analysis of real-time systems using time Petri nets, Vol. 30, IEEE Transactions on Systems, Man, and Cybernetics - Part B, pp. $725-736$.

$\mathrm{Xu}, \mathrm{D} ., \mathrm{He}, \mathrm{X}$. and Deng, Y. (2002). Compositional schedulability analysis of real-time systems using time Petri nets, IEEE Trans. Soft. Engineering 28(10): 984996.

Yoneda, T. and Schlingloff, B.-H. (1997). Efficient verification of parallel real-time systems, Formal Methods in System Design, Vol. 11, pp. 187-215. 